Dharmendra Kumar*

\title{
Positive Solution to Singular Elliptic Problems with Subcritical nonlinearities
}

https://doi.org/10.1515/msds-2019-0007

Received July 25, 2019; accepted December 20, 2019

Abstract: In this paper, we study the existence of a non-trivial weak solution to the following singular elliptic equations with subcritical nonlinearities:

$$
\left\{\begin{array}{l}
-\operatorname{div}\left(|x|^{-2 \beta} \nabla u\right)-\mu \frac{f(x) u}{|x|^{2(\beta+1)}}=\frac{\lambda g(x)}{u^{\theta}}+h(x) u^{p} \text { in } \Omega, \\
u>0 \text { in } \Omega, \\
u=0 \text { on } \partial \Omega,
\end{array}\right.
$$

where $\Omega \subset \mathbb{R}^{N}$ is an open bounded domain with $C^{1}$ boundary, $\theta, \lambda>0,0<\beta<\frac{N-2}{2}, 0<p<1,0<\mu<$ $\left(\frac{N-2(\beta+1)}{2}\right)^{2}, N \geq 3,0 \in \Omega$ and $0 \leq f, g, h \in L^{\infty}(\Omega)$. We show that there exists a solution $u \in H_{0}^{1}\left(\Omega,|x|^{-2 \beta}\right) \cap$ $L^{\infty}(\Omega)$ to this problem.

Keywords: Nonlinear elliptic equation, Hardy potential, positive solution

MSC: Primary 35J05, 35J25; Secondary 46E35

\section{Introduction}

Let $\Omega \subset \mathbb{R}^{N}$ be an open bounded domain with $C^{1}$ boundary, $0 \in \Omega$. We consider the following singular elliptic equations with subcritical nonlinearities:

$$
\left\{\begin{array}{l}
-\operatorname{div}\left(|x|^{-2 \beta} \nabla u\right)-\mu \frac{f(x) u}{|x|^{2(\beta+1)}}=\frac{\lambda g(x)}{u^{\theta}}+h(x) u^{p} \text { in } \Omega, \\
u>0 \text { in } \Omega, \\
u=0 \text { on } \partial \Omega,
\end{array}\right.
$$

under assumptions $\theta, \lambda>0,0<\beta<\frac{N-2}{2}, 0<p<1,0<\mu<\left(\frac{N-2(\beta+1)}{2}\right)^{2}, N \geq 3$, and $0 \leq f, g, h \in$ $L^{\infty}(\Omega)$. We prove existence of (1.1) in $H_{0}^{1}\left(\Omega,|x|^{-2 \beta}\right) \cap L^{\infty}(\Omega)$. Furthermore, a solution of (1.1) is a function $u$ in $W_{0}^{1,1}\left(\Omega,|\chi|^{-2 \beta}\right)$ with the following:

$$
\forall \Omega^{\prime} \subset \subset \Omega, \exists c_{\Omega^{\prime}}: u \geq c_{\Omega^{\prime}}>0 \text { in } \Omega^{\prime},
$$

and

$$
\int_{\Omega}|x|^{-2 \beta} \nabla u \nabla \varphi-\mu \int_{\Omega} f(x) \frac{u \varphi}{|x|^{2(\beta+1)}}=\int_{\Omega} \frac{g(x) \varphi}{u^{\theta}}+\int_{\Omega} h(x) u^{p} \varphi, \quad \forall \varphi \in C_{0}^{1}(\Omega) .
$$

We pointed out that the right hand side is well defined by (1.2) since $\varphi$ has compact support.

\footnotetext{
`Corresponding Author: Dharmendra Kumar: Indian Institute of Technology Gandhinagar, Gujarat, India - 382355, E-mail:
} k.dharmendra@iitgn.ac.in, dharamsambey90@gmail.com 
The problem of the form (1.1) arise in certain problems in fluid mechanics, chemical heterogenous catalysts, pseudo plastic flow and non-Newtonian fluids ( see $[3,9,13,14,17])$. Simpler form of the problem (1.1) is of the type

$$
-\operatorname{div}(M(x) \nabla u)=h(x, u),
$$

where $M(x)=|x|^{-2 \beta}$ and $h(x, s)$ is singular for $s=0$ and $x=0$. Equation (1.4) with $M(x)$ is identity matrix, has been widely studied by many authors in the past, see, for instance $[2,5-7,12,16]$ and the reference therein. When $h(x, s)$ that does not depend on $x$, Crandall, Rabinowitz and Tartar in [7], showed the existence and continuity properties of the solution of (1.4). In [12], it has been shown that the existence of the solution to (1.4), for $h(x, u)=\frac{f(x)}{u^{\gamma}}$, where $f$ is a continuous function. They showed that the solution $u \in H_{0}^{1}(\Omega)$ if and only if $\gamma<3$ and that, if $\gamma>1$, solution $u \notin C^{1}(\bar{\Omega})$. For, $h(x, u)=f(x) g(u)$, Lair and Shaker [10, 11] studied the problem (1.4) , and by Zhang and Cheng, see [19], using the first eigenfunction of the Laplacian in $\Omega$.

The present work is more close to results in $[1,8]$, although we generalised the results for sublinearities, i.e. for the case $0<p<1$ [1, Theorem 2.4]. The aim of this paper is to show the existence to (1.1) for sublinearities. To obtain a solution to (1.1), we use the similar techniques as used in [1, 2]. The organisation of this paper is as follows. Section 2 deals with some preliminaries, basic facts which are used in the main result. Section 3 is devoted to the proof of the main theorem.

The main result of this paper is the following theorem, which we will prove in the next section.

Theorem 1.1. Assume (1.1) with $0<p<1, \theta, \lambda>0,0<\beta<\frac{N-2}{2}, 0<\mu<\left(\frac{N-2(\beta+1)}{2}\right)^{2}$, and $0 \leq f, g, h \in$ $L^{\infty}(\Omega)$. Then $\exists \Lambda \in(0, \infty)$ such that for $\lambda \in(0, \Lambda), \exists$ a solution $u$ in $H_{0}^{1}\left(\Omega,|x|^{-2 \beta}\right)$ to (1.1).

Moreover

- If $0<\theta<1$, then $u \in H_{0}^{1}\left(\Omega,|x|^{-2 \beta}\right) \cap L^{\infty}(\Omega)$.

\section{Auxiliary results}

Let $n \in \mathbb{N}$, let $g_{n}(x)=\min \{g(x), n\}$. To prove the existence results we consider the following approximate problem:

$$
\left\{\begin{array}{l}
-\operatorname{div}\left(|x|^{-2 \beta} \nabla u_{n}\right)-\mu \frac{f(x) u_{n}}{|x|^{2(\beta+1)}+\frac{1}{n}}=\frac{\lambda g_{n}(x)}{\left(u_{n}+\frac{1}{n}\right)^{\theta}} \text { in } \Omega, \\
u_{n}>0 \text { in } \Omega \\
u_{n}=0 \text { on } \partial \Omega
\end{array}\right.
$$

Let us recall the following Caffarelli-Kohn-Nirenberg inequality and a compact embedding theorem (which is an extension of the classical Rellich-Kondrachov compactness theorem).

Proposition 2.1. Caffarelli-Kohn-Nirenberg inequality [4]: There is a constant $C_{\gamma, \beta}>0$ such that

$$
\left(\int_{\mathbb{R}^{N}}|x|^{-2 \star \beta}|u|^{2 *}\right)^{2 / 2 \star} \leq C_{\gamma, \beta} \int_{\mathbb{R}^{N}}|x|^{-2 \gamma}|\nabla u|^{2},
$$

for all $u \in C_{0}^{\infty}\left(\mathbb{R}^{N}\right)$, where

$$
-\infty<\gamma<\frac{N-2}{2}, \quad \beta \leq \gamma<\beta+1, \quad 2 \star=\frac{2 N}{N-2 d}, d=\gamma+1-\beta .
$$


Let $H_{0}^{1}\left(\Omega,|x|^{-2 \beta}\right)$ be the completion of $C_{0}^{\infty}\left(\mathbb{R}^{N}\right)$, with respect to the following weighted norm $\|\cdot\|$ defined by

$$
\|u\|=\left(\int_{\Omega}|x|^{-2 \beta}|\nabla u|^{2}\right)^{1 / 2} .
$$

From the boundedness of $\Omega$ and the standard approximation arguments, it can be shown that (2.2) holds for any $u \in H_{0}^{1}\left(\Omega,|x|^{-2 \beta}\right)$ in the sense:

$$
\left(\int_{\Omega}|x|^{-\beta q}|u|^{q}\right)^{2 / q} \leq C_{\beta} \int_{\Omega}|x|^{-2 \gamma}|\nabla u|^{2},
$$

for $1 \leq q \leq 2 \star$.

Proposition 2.2. General Hardy-Sobolev inequality: There is a constant $C_{\beta, N, 2}>0$ such that

$$
\left(\int_{\Omega}|x|^{-2(\beta+1)}|u|^{2}\right) \leq C_{\beta, N, 2}^{-1} \int_{\Omega}|x|^{-2 \beta}|\nabla u|^{2},
$$

for all $u \in H_{0}^{1}\left(\Omega,|x|^{-2 \beta}\right)$, where

$$
-\infty<\beta<\frac{N-2}{2}, C_{\beta, N, 2}=\left(\frac{N-2(\beta+1)}{2}\right)^{2} .
$$

Moreover, $C_{\beta, N, 2}^{-1}$ is optimal and it is not achieved.

Let $L^{q}\left(\Omega,|x|^{-\beta q}\right)$ be the weighted $L^{q}$ space with weighted norm defined by

$$
\|u\|_{\beta, q}:=\left(\int_{\Omega}|x|^{-\beta q}|u|^{q}\right)^{1 / q} .
$$

then (2.5) can be written as

$$
\|u\|_{\beta, q} \leq C\|u\|, \quad \forall u \in H_{0}^{1}\left(\Omega,|x|^{-2 \beta}\right),
$$

where $C>0$ denotes a universal constant, which may change its value from line to line.

Proposition 2.3. Compact imbedding theorem[18]: Suppose that $\Omega \subset \mathbb{R}^{N}$ is an open bounded domain with $C^{1}$ boundary and $0 \in \Omega, N>2,-\infty<\gamma<\frac{N-2}{2}, \beta \leq \gamma \leq(1+\beta)$.

For $1 \leq q \leq 2 \star$, the imbedding $H_{0}^{1}\left(\Omega,|x|^{-2 \beta}\right) \hookrightarrow L^{q}\left(\Omega,|x|^{-\gamma q}\right)$ is continuous.

For $1 \leq q<2 *$, the imbedding $H_{0}^{1}\left(\Omega,|x|^{-2 \beta}\right) \hookrightarrow L^{q}\left(\Omega,|x|^{-\gamma q}\right)$ is compact.

Lemma 2.4. For $C_{\beta, N, 2}^{-1}\|f\|_{L^{\infty}(\Omega)}<\frac{1}{\mu}$ where $0<\beta<\frac{N-2}{2}, C_{\beta, N, 2}=\left(\frac{N-2(\beta+1)}{2}\right)^{2}$. Then Problem (2.1) has a nonnegative solution in $H_{0}^{1}\left(\Omega,|x|^{-2 \beta}\right) \cap L^{\infty}(\Omega)$.

Proof. Let $n \in \mathbb{N}$ be a fixed natural no and $\omega$ be a function in $L^{2}\left(\Omega,|x|^{-2 \beta}\right)$. By the Lax-Milgram Theorem, the following problem has a unique solution $u$ in $H_{0}^{1}\left(\Omega,|x|^{-2 \beta}\right) \cap L^{\infty}(\Omega)$ :

$$
\left\{\begin{array}{l}
-\operatorname{div}\left(|x|^{-2 \beta} \nabla u\right)-\mu \frac{f(x) u}{|x|^{2(\beta+1)+\frac{1}{n}}}=\frac{g_{n}(x)}{\left(|\omega|+\frac{1}{n}\right)^{\theta}} \text { in } \Omega, \\
u>0 \text { in } \Omega, \\
u=0 \text { on } \partial \Omega,
\end{array}\right.
$$


because the operator is coercive due to the assumptions on $\mu$ and Proposition 2.2. Now, for any $u \in L^{2}\left(\Omega,|x|^{-2(\beta+1)}\right)$, we define the mapping

$$
T: L^{2}\left(\Omega,|x|^{-2(\beta+1)}\right) \rightarrow H_{0}^{1}\left(\Omega,|x|^{-2 \beta}\right) \cap L^{\infty}(\Omega),
$$

as follows:

$$
T(u)=\omega .
$$

Let us take $u$ as a test function, we have

$$
\int_{\Omega}|x|^{-2 \beta}|\nabla u|^{2}-\mu \int_{\Omega} \frac{f(x) u^{2}}{|x|^{2(\beta+1)}+\frac{1}{n}}=\int_{\Omega} \frac{g_{n}(x) u}{\left(|\omega|+\frac{1}{n}\right)^{\theta}} .
$$

and so

$$
\int_{\Omega}|x|^{-2 \beta}|\nabla u|^{2}-\mu \int_{\Omega} \frac{f(x) u^{2}}{|x|^{2(\beta+1)}+\frac{1}{n}} \leq n^{\theta+1} \int_{\Omega} u,
$$

Since $g_{n}(x)=\min \{g(x), n\}$, so $g_{n}(x) \leq n$, this implies that

$$
\frac{g_{n}(x) u}{\left(|\omega|+\frac{1}{n}\right)^{\theta}} \leq \frac{n u}{(n|\omega|+1)^{\theta}} \times n^{\theta} \leq n^{\theta+1} u,
$$

The General Hardy - Sobolev inequality on the left-hand side and Hölder inequality on the right-hand side implies that

$$
\left(C_{\beta, N, 2}-\mu\|f\|_{\infty}\right) \int_{\Omega}|x|^{-2(\beta+1)}|u|^{2} \leq C n^{\theta+1}\left(\int_{\Omega} u^{2}\right)^{1 / 2},
$$

for some constant $C$ independent of $u$.

This is equivalent to

$$
\left(C_{\beta, N, 2}-\mu\|f\|_{\infty}\right) \int_{\Omega}|x|^{-2(\beta+1)}|u|^{2} \leq C n^{\theta+1}|\Omega|^{2(\beta+1)}\left(\int_{\Omega}|x|^{-2(\beta+1)} u^{2}\right)^{1 / 2},
$$

where $|\Omega|$ denotes the diameter of $\Omega$.

This implies that

$$
\leq C n^{\theta+1}|\Omega|^{(\beta+1)}\left(\int_{\Omega}|x|^{-2(\beta+1)} u^{2}\right)^{1 / 2},
$$

i.e. the ball of radius $C n^{\theta+1}$ is invariant under $T$. Using compact imbedding, it can be shown that $T$ is both continuous and compact on $L^{2}\left(\Omega,|x|^{-2(\beta+1)}\right)$. So by Schauder's fixed point theorem, $\exists u_{n} \in H_{0}^{1}\left(\Omega,|x|^{-2 \beta}\right)$ such that $u_{n}=T\left(u_{n}\right)$, i.e., $u_{n}$ solves

$$
\left\{\begin{array}{l}
-\operatorname{div}\left(|x|^{-2 \beta} \nabla u_{n}\right)-\mu \frac{f(x) u_{n}}{|x|^{2(\beta+1)}+\frac{1}{n}}=\frac{g_{n}(x)}{\left(u_{n}+\frac{1}{n}\right)^{\theta}} \text { in } \Omega, \\
u_{n}>0 \text { in } \Omega \\
u_{n}=0 \text { on } \partial \Omega
\end{array}\right.
$$

Since $\frac{g_{n}(x)}{\left(\omega+\frac{1}{n}\right)^{\theta}} \geq 0$ a.e., so the maximum principle implies that $u_{n} \geq 0$ and hence $u_{n}$ be a solution of (2.1). By the result of [15], $u_{n}$ belongs to $L^{\infty}(\Omega)$, because of the right-hand side of (2.10) belongs to $L^{\infty}(\Omega)$. 


\section{Proof of the theorem 1.1}

Proof. Consider the following Dirichlet problems

$$
u_{n} \in H_{0}^{1}\left(\Omega,|x|^{-2 \beta}\right):-\operatorname{div}\left(|x|^{-2 \beta} \nabla u_{n}\right)-\mu \frac{f(x) u_{n}}{|x|^{2(\beta+1)}+\frac{1}{n}}=\frac{\lambda g_{n}(x)}{\left(u_{n}+\frac{1}{n}\right)^{\theta}}+h(x) u_{n}^{p} \text { in } \Omega .
$$

To prove the existence of a solution $u_{n}$ of (3.1), we apply Sattinger monotone iteration.

We first obtain a subsolution:

By Lemma(2.4) there exists a weak solution $\underline{u_{n}}$ of the following Dirichlet problem

$$
\underline{u_{n}} \in H_{0}^{1}\left(\Omega,|x|^{-2 \beta}\right):-\operatorname{div}\left(|x|^{-2 \beta} \nabla \underline{u_{n}}\right)-\mu \frac{f(x) \underline{u_{n}}}{|x|^{2(\beta+1)}+\frac{1}{n}}=\frac{\lambda g_{n}(x)}{\left(\underline{u_{n}}+\frac{1}{n}\right)^{\theta}} \text { in } \Omega,
$$

so $u_{n}$ solves:

$$
\underline{u_{n}} \in H_{0}^{1}\left(\Omega,|x|^{-2 \beta}\right):-\operatorname{div}\left(|x|^{-2 \beta} \nabla \underline{u_{n}}\right)-\mu \frac{f(x) \underline{u}_{n}}{|x|^{2(\beta+1)}+\frac{1}{n}}=\frac{\lambda g_{n}(x)}{\left(\underline{u_{n}}+\frac{1}{n}\right)^{\theta}} \text { in } \Omega .
$$

Thus

$$
\underline{u_{n}} \in H_{0}^{1}\left(\Omega,|x|^{-2 \beta}\right):-\operatorname{div}\left(|x|^{-2 \beta} \nabla \underline{u_{n}}\right)-\mu \frac{f(x) \underline{u}_{n}}{|x|^{2(\beta+1)}+\frac{1}{n}} \leq \frac{\lambda g_{n}(x)}{\left(\underline{u_{n}}+\frac{1}{n}\right)^{\theta}}+h(x){\underline{u_{n}}}^{p} \text { in } \Omega,
$$

i.e $u_{n}$ is a subsolution to the problem (3.1).

Now, we construct a supersolution of (3.1):

Let $t \geq Y_{\lambda}$ (yet have to determine) and consider the following Dirichlet problems $\overline{u_{n, t}}: \overline{u_{n, t}}$ is the solution of

$$
\overline{u_{n, t}} \in H_{0}^{1}\left(\Omega,|x|^{-2 \beta}\right):-\operatorname{div}\left(|x|^{-2 \beta} \nabla \overline{u_{n, t}}\right)-\mu \frac{f(x) \overline{u_{n, t}}}{|x|^{2(\beta+1)}+\frac{1}{n}}=\frac{t}{\left(\overline{u_{n, t}}+\frac{1}{n}\right)^{\theta}},
$$

then (see [2]) $\exists c_{0}>0$ such that

$$
\left\|\overline{u_{n, t}}\right\|_{L^{\infty}(\Omega)} \leq c_{0} t^{\frac{1}{\theta+1}} .
$$

Then the maximum principle implies that $\overline{u_{n, t}} \geq 0$ so that $\overline{u_{n, t}}$ solves

$$
-\operatorname{div}\left(|x|^{-2 \beta} \nabla \overline{u_{n, t}}\right)-\mu \frac{f(x) \overline{u_{n, t}}}{|x|^{2(\beta+1)}+\frac{1}{n}}=\frac{t}{\left(\overline{u_{n, t}}+\frac{1}{n}\right)^{\theta}} .
$$

We show that for some $t>0$,

$$
\frac{t}{\left(\frac{1}{n}+\overline{u_{n, t}}\right)^{\theta}} \geq \frac{\lambda g(x)}{\left(\frac{1}{n}+\overline{u_{n, t}}\right)^{\theta}}+h(x) \overline{u_{n, t}} p
$$

i.e;

$$
t \geq \lambda g(x)+h(x) \overline{u_{n, t}} p\left(\frac{1}{n}+\overline{u_{n, t}}\right)^{\theta} .
$$

The above inequality is true if $t$ satisfy the following

$$
t \geq \lambda\|g\|_{\infty}+\|h\|_{\infty}\left[c_{0} t^{\frac{1}{\theta+1}}\right]^{p}\left(\frac{1}{n}+c_{0} t^{\frac{1}{\theta+1}}\right)^{\theta} .
$$

Suppose $Y_{\lambda}$ be such that

$$
Y_{\lambda}=\lambda\|g\|_{\infty}+2^{\theta}\|h\|_{\infty} c_{0}^{p+\theta} Y_{\lambda}^{\frac{p+\theta}{\theta+1}} .
$$

Let $F\left(Y_{\lambda}\right)=Y_{\lambda}-\lambda\|g\|_{\infty}-2^{\theta}\|h\|_{\infty} c_{0}^{p+\theta} Y_{\lambda}^{\frac{p+\theta}{\theta+1}}$.

Then

$$
F(0)=-\lambda\|g\|_{\infty}<0 .
$$


Now, let

$$
G\left(Y_{\lambda}\right)=Y_{\lambda}^{1-\frac{p+\theta}{\theta+1}}-2^{\theta}\|h\|_{\infty} c_{0}^{p+\theta}-\lambda\|g\|_{\infty} Y_{\lambda}^{-\frac{p+\theta}{\theta+1}}
$$

Then let $Y_{\lambda} \rightarrow \infty$,

$$
\Rightarrow \quad G\left(Y_{\lambda}\right) \rightarrow \infty \text {, i.e; }
$$

$\exists N \in \mathbb{N}$ such that $G\left(Y_{\lambda}\right)>1, \quad \forall Y_{\lambda} \geq N$,

$$
\Rightarrow G(N)>1 .
$$

Now,

$$
F\left(Y_{\lambda}\right)=Y_{\lambda}^{\frac{p+\theta}{\theta+1}} G\left(Y_{\lambda}\right) .
$$

Hence

$$
F(N)=N^{\frac{p+\theta}{\theta+1}} G(N)>1 \cdot N^{\frac{p+\theta}{\theta+1}}>0 .
$$

By Mean Value Theorem, $\exists Y_{\lambda}$ such that $0<Y_{\lambda}<N$ and $F\left(Y_{\lambda}\right)=0$. That is there exists $\Lambda>0$ such that for $0<\lambda<\Lambda$, there exists the solution of the equation (3.11). Therefore, we only have to prove that if $n$ is large enough, we have

$$
\lambda\|g\|_{\infty}+2^{\theta}\|h\|_{\infty} c_{0}^{p+\theta} Y_{\lambda}^{\frac{p+\theta}{\theta+1}} \geq \lambda\|g\|_{\infty}+\|h\|_{\infty} c_{0}^{p} Y_{\theta}^{\frac{p}{\theta+1}}\left(\frac{1}{n}+c_{0} Y_{\lambda}^{\frac{1}{\theta+1}}\right)^{\theta}
$$

thus,

$$
2^{\theta} c_{0}^{\theta} Y_{\lambda}^{\frac{\theta}{\theta+1}} \geq\left(\frac{1}{n}+c_{0} Y_{\lambda}^{\frac{1}{\theta+1}}\right)^{\theta}
$$

this will hold if $\frac{1}{n} \leq c_{0} Y_{\lambda}^{\frac{1}{\theta+1}}$ and this latter inequality true for $n$ large.

Since $Y_{\lambda}$ independent of $n$. Thus we proved that for $Y \geq Y_{\lambda}, \overline{u_{n, t}}$ is a super solution to the problem (3.1).

We show that $u_{n} \leq \overline{u_{n, t}}$ :

Define

$$
L(v)=-\operatorname{div}\left(|x|^{-2 \beta} \nabla v\right)-\mu \frac{f(x) v}{|x|^{2(\beta+1)}+\frac{1}{n}} .
$$

Next aim to show $\underline{u_{n}} \leq \overline{u_{n, t}}$. Indeed

$L\left(\underline{u_{n}}-\overline{u_{n, t}}\right)=$

$$
\begin{aligned}
& -\operatorname{div}\left(|x|^{-2 \beta} \nabla \underline{u_{n}}\right)-\mu \frac{f(x) \underline{u_{n}}}{|x|^{2(\beta+1)}+\frac{1}{n}}+\operatorname{div}\left(|x|^{-2 \beta} \nabla \overline{u_{n, t}}\right)+\mu \frac{f(x) \overline{u_{n, t}}}{|x|^{2(\beta+1)}+\frac{1}{n}} \\
& =\frac{\lambda g_{n}(x)}{\left(\frac{1}{n}+\left|\underline{u_{n}}\right|\right)^{\theta}}-\frac{t}{\left(\frac{1}{n}+\overline{u_{n, t}}\right)^{\theta}},
\end{aligned}
$$

implies

$$
\left\langle L\left(\underline{u_{n}}-\overline{u_{n, t}}\right),\left(\underline{u_{n}}-\overline{u_{n, t}}\right)^{+}\right\rangle=\int_{\Omega}\left[\frac{\lambda g_{n}(x)}{\left(\frac{1}{n}+\left|\underline{u_{n}}\right|\right)^{\theta}}-\frac{t}{\left(\frac{1}{n}+\overline{u_{n, t}}\right)^{\theta}}\right]\left(\underline{u_{n}}-\overline{u_{n, t}}\right)^{+} .
$$

Since $g_{n}(x)=\min \{n, g(x)\} \leq g(x) \leq\|g(x)\|_{L^{\infty}(\Omega)}$, so we have

$$
\left\langle L\left(\underline{u_{n}}-\overline{u_{n, t}}\right),\left(\underline{u_{n}}-\overline{u_{n, t}}\right)^{+}\right\rangle \leq \int_{\Omega}\left[\frac{\lambda\|g\|_{L^{\infty}}}{\left(\frac{1}{n}+\left|\underline{u_{n}}\right|\right)^{\theta}}-\frac{t}{\left(\frac{1}{n}+\overline{u_{n, t}}\right)^{\theta}}\right]\left(\underline{u_{n}}-\overline{u_{n, t}}\right)^{+}
$$




$$
\begin{aligned}
& =\int_{\Omega}\left[\frac{\lambda\|g\|_{L^{\infty}}}{\left(\frac{1}{n}+\left|\underline{u_{n}}\right|\right)^{\theta}}-\frac{\lambda\|g\|_{L^{\infty}}}{\left(\frac{1}{n}+\overline{u_{n, t}}\right)^{\theta}}\right]\left(\underline{u_{n}}-\overline{u_{n, t}}\right)^{+} \\
& +\int_{\Omega}\left[\frac{\lambda\|g\|_{L^{\infty}}}{\left(\frac{1}{n}+\left|\underline{u_{n}}\right|\right)^{\theta}}-\frac{t}{\left(\frac{1}{n}+\overline{u_{n, t}}\right)^{\theta}}\right]\left(\underline{u_{n}}-\overline{u_{n, t}}\right)^{+} \\
& =\lambda \int_{\left\{\underline{u_{n}} \geq \overline{u_{n, t}}\right\}}\|g\|_{L^{\infty}}\left[\frac{1}{\left(\frac{1}{n}+\left|\underline{u_{n}}\right|\right)^{\theta}}-\frac{1}{\left(\frac{1}{n}+\overline{u_{n, t}}\right)^{\theta}}\right]\left(\underline{u_{n}}-\overline{u_{n, t}}\right) \\
& +\int_{\left\{\underline{u_{n}} \geq \overline{u_{n, t}}\right\}} \frac{1}{\left(\frac{1}{n}+\left|\overline{u_{n, t}}\right|\right)^{\theta}}\left[\lambda\|g\|_{L^{\infty}}-t\right]\left(\underline{u_{n}}-\overline{u_{n, t}}\right) .
\end{aligned}
$$

Note that, in the last line, the first integral is negative because the real function $\frac{1}{\left(\frac{1}{n}+l\right)^{\theta}}$ is decreasing if $l>0$ and the second integral is negative because $t \geq Y_{\lambda}$ and by the definition (3.11), $Y_{\lambda} \geq \lambda\|g\|_{\infty}$.

Next we apply Sattinger Method:

$$
w(s):=\frac{\lambda g(x)}{\left(\frac{1}{n}+s\right)^{\theta}}+h(x) s^{p}+n^{\theta+1} \lambda \theta s, \quad 0<s<\infty .
$$

It can be shown that function $w(s)$ is increasing.

Now the classical Amann-Sattinger method will give the existence of $u_{n}$ solution of

and such that

$$
\begin{gathered}
u_{n} \in H_{0}^{1}\left(\Omega,|x|^{-2 \beta}\right):-\operatorname{div}\left(|x|^{-2 \beta} \nabla u_{n}\right)-\mu \frac{f(x) u_{n}}{|x|^{2(\beta+1)}+\frac{1}{n}}+n^{\theta+1} \lambda \theta u_{n} \\
=\frac{\lambda g(x)}{\left(\frac{1}{n}+u_{n}\right)^{\theta}}+h(x)\left(u_{n}^{+}\right)^{p}+n^{\theta+1} \lambda \theta u_{n},
\end{gathered}
$$

$$
\underline{u_{n}} \leq u_{n} \leq \omega_{n, t} \leq c_{0} t^{\frac{1}{\theta+1}} .
$$

also using [2], it can be shown that the sequence $\left\{\underline{u_{n}}\right\}$ is increasing with respect to $n, \underline{u_{n}}>0$ in $\Omega$ and for every $\Omega^{\prime} \subset \subset \Omega, \exists c_{\Omega^{\prime}}$ (independent of $n$ ) such that

$$
\underline{u_{n}}(x) \geq \omega_{1}(x) \geq c_{\Omega^{\prime}}>0, \quad \forall x \in \Omega^{\prime}, n \in \mathbb{N} .
$$

We will use $u_{n}$ as a test function in the following equation:

$$
u_{n} \in H_{0}^{1}\left(\Omega,|x|^{-2 \beta}\right):-\operatorname{div}\left(|x|^{-2 \beta} \nabla u_{n}\right)-\mu \frac{f(x) u_{n}}{|x|^{2(\beta+1)}+\frac{1}{n}}=\frac{\lambda g_{n}(x)}{\left(u_{n}+\frac{1}{n}\right)^{\theta}}+h(x) u_{n}^{p} .
$$

Indeed,

$$
\begin{gathered}
\int_{\Omega}|x|^{-2 \beta} \nabla u_{n} \nabla u_{n}-\frac{\mu}{2} \int_{\Omega} \frac{f(x) u_{n}^{2}}{|x|^{2(\beta+1)}+\frac{1}{n}}=\int_{\Omega} \frac{\lambda g(x) u_{n}}{\left(\frac{1}{n}+u_{n}\right)^{\theta}}+\int_{\Omega} h(x)\left(u_{n}^{+}\right)^{p} u_{n}, \\
\left(1-\mu\|f\|_{\infty} C_{\beta, N, 2}^{-1}\right) \int_{\Omega}|x|^{-2 \beta}\left|\nabla u_{n}\right|^{2} d x \leq \lambda\|g\|_{L^{\infty}(\Omega)} \int_{\Omega} u_{n}^{1-\theta}+\|h\|_{\infty} \int_{\Omega}\left|u_{n}\right|^{p+1},
\end{gathered}
$$

(3.23) implies that $\left\{u_{n}\right\}$ is bounded in $H_{0}^{1}\left(\Omega,|x|^{-2 \beta}\right)$. Then $\exists$ a subsequence $\left\{u_{n}\right\}$ (still denoted by $\left\{u_{n}\right\}$ ) of sequence $\left\{u_{n}\right\}$ and $u \in H_{0}^{1}\left(\Omega,|x|^{-2 \beta}\right) \cap L^{\infty}(\Omega)$ such that

$$
u_{n} \rightarrow u
$$

weakly in $H_{0}^{1}\left(\Omega,|x|^{-2 \beta}\right)$ and strongly in $L^{2}\left(\Omega,|x|^{-2 \beta}\right)$,

$$
u_{n} \rightarrow u \text { a.e. in } \Omega \text {, }
$$




$$
\nabla u_{u} \rightarrow \nabla u \text { weakly in } L^{2}\left(\Omega,|x|^{-2 \beta}\right) .
$$

So we have,

$$
\lim _{n \rightarrow \infty} \int_{\Omega}|x|^{-2 \beta} \nabla u_{n} \nabla \varphi=\int_{\Omega}|x|^{-2 \beta} \nabla u \nabla \varphi, \quad \forall \phi \in H_{0}^{1}\left(|x|^{-2 \beta}\right) .
$$

Furthermore, we have, for $\phi \in H_{0}^{1}\left(\Omega,|x|^{-2 \beta}\right)$,

$$
0 \leq \frac{\lambda g(x) \phi}{\left(\frac{1}{n}+u_{n}\right)^{\theta}} \leq \frac{\lambda\|g\|_{\infty} \phi}{\left(c_{\Omega^{\prime}}\right)^{\theta}},
$$

since, recalling (3.23) and (3.24), $u_{n} \geq \underline{u_{n}} \geq \underline{u_{1}} \geq c_{\Omega^{\prime}}$ and $0<\theta \leq 1$.

By the Lebesgue dominated convergence theorem, we have

$$
\lim _{n \rightarrow \infty} \int_{\Omega} \frac{\lambda g(x) \phi}{\left(\frac{1}{n}+u_{n}\right)^{\theta}}=\lambda \int_{\Omega} \frac{g(x) \phi}{u^{\theta}} .
$$

Remark 3.1. As in [1], the existence to (1.1) still holds for $p>1$.

Remark 3.2. The similar proof as in the Theorem 1.1, will work to treat the following semilinear elliptic problem:

$$
\left\{\begin{array}{l}
-\operatorname{div}(M(x) \nabla u)-\mu \frac{g(x) u}{|x|^{2}}=\frac{\lambda f(x)}{u^{\gamma}}+h(x) u^{p} \text { in } \Omega, \\
u>0 \text { in } \Omega, \\
u=0 \text { on } \partial \Omega,
\end{array}\right.
$$

where $\Omega \subset \mathbb{R}^{N}$ is an open bounded subset, $N \geq 3 ; 0 \in \Omega, 0 \leq f, g, h \in L^{\infty}(\Omega)$. Under assumptions $\gamma, 0<p<$ $1, \lambda>0$ small and $M$ be bounded elliptic matrix, i.e, $\exists 0<\alpha \leq \beta$ such that $\alpha|\eta|^{2} \leq\langle M(x) \eta, \eta\rangle,|M(x)| \leq \beta$. As in Theorem 1.1. $\exists \Lambda \in(0, \infty)$ such that for $\lambda \in(0, \Lambda) \exists$ a solution $u$, strictly positive in $\Omega$ of (3.34), in the following sense that

$$
\int_{\Omega} M(x) \nabla u \nabla \varphi-\mu \int_{\Omega} \frac{f(x) u \varphi}{\left(|x|^{2}\right.}=\lambda \int_{\Omega} \frac{g(x) \varphi}{u^{\gamma}}+\int_{\Omega} h(x) u^{p} \varphi, \quad \forall \varphi \in H_{0}^{1}(\Omega),
$$

where $\Omega^{\prime}$ is an arbitrary open subset of $\Omega$, such that $\overline{\Omega^{\prime}} \subset \Omega$.

Moreover if $0<\gamma<1$, then $u \in H_{0}^{1}(\Omega) \cap L^{\infty}(\Omega)$. For the sake of brevity, we omit the proof.

Remark 3.3. [8] The similar proof as in the Theorem 1.1, will work to treat the following semilinear elliptic problem:

$$
\left\{\begin{array}{l}
-\operatorname{div}\left(M(\xi) \nabla_{\mathbb{H}^{n}} u\right)-\mu \frac{g(\xi) u}{\left(|z|^{4}+t^{2}\right)^{1 / 2}}=\frac{\lambda f(\xi)}{u^{\gamma}}+h(\xi) u^{p} \text { in } \Omega, \\
u>0 \text { in } \Omega, \\
u=0 \text { on } \partial \Omega,
\end{array}\right.
$$

where $\Omega \subset \mathbb{H}^{n}$ is an open bounded subset, $N \geq 3 ; 0 \in \Omega, 0 \leq f, g, h \in L^{\infty}(\Omega)$. Under assumptions $\gamma, 0<p<$ $1, \lambda>0$ small and $M$ be bounded elliptic matrix, i.e, $\exists 0<\alpha \leq \beta$ such that $\alpha|\eta|^{2} \leq\langle M(\xi) \eta, \eta>,|M(\xi)| \leq \beta$. As in Theorem 1.1. $\exists \Lambda \in(0, \infty)$ such that for $\lambda \in(0, \Lambda) \exists$ a solution $u$, strictly positive in $\Omega$ of (3.36), in the following sense that

$$
\int_{\Omega} M(\xi) \nabla_{\mathbb{H}^{n}} u \nabla_{\mathbb{H}^{n}} \varphi-\mu \int_{\Omega} \frac{f(\xi) u \varphi}{\left(|z|^{4}+t^{2}\right)^{1 / 2}}=\lambda \int_{\Omega} \frac{g(\xi) \varphi}{u^{\gamma}}+\int_{\Omega} h(\xi) u^{p} \varphi,
$$

$\forall \varphi \in H_{0}^{1}\left(\Omega, \mathbb{H}^{n}\right)$, where $\Omega^{\prime}$ is an arbitrary open subset of $\Omega$, such that $\overline{\Omega^{\prime}} \subset \Omega$.

Moreover if $0<\gamma<1$, then $u \in H_{0}^{1}\left(\Omega, \mathbb{H}^{n}\right) \cap L^{\infty}(\Omega)$. For the sake of brevity, we omit the proof. 
Acknowledgements: The author thank the anonymous referee for carefully reading the manuscript and for his/her helpful comments and suggestions. The author also thanks Dr Jagmohan Tyagi for suggesting this problem and IIT Gandhinagar for the financial support.

\section{References}

[1] L. Boccardo, A Dirichlet problem with singular and supercritical nonlinearities, Nonlinear Anal. 75 (12)(2012), 4436-4440.

[2] L. Boccardo, L. Orsina, Semilinear elliptic equations with singular nonlinearities, Calc. Var. Partial Differential Equations, 37 (2010), 363-380.

[3] C.M. Brauner, B. Nicolaenko, On nonlinear eigenvalue problems which extend into free boundaries problems. In: Nonlinear Eigenvalue Problems and Bifurcation, Lecture Notes in Math, 782(1980), 61-100.

[4] L. Caffarelli, R. Kohn and L. Nirenberg, First order interpolation inequality with weights, Compositio Math. 53 (1984) 259275.

[5] A. Canino, M. Degiovanni, A variational approach to a class of singular semilinear elliptic equations, J. Convex Anal. 11 (2004), 147-162.

[6] M.M. Coclite, G. Palmieri, On a singular nonlinear Dirichlet problem, Comm. Partial Differential Equations 14(1989), 13151327.

[7] M. G. Crandall, P. H. Rabinowitz, Luc C Tartar, On a Dirichlet problem with a singular nonlinearity, Comm. Partial Differ. Equ. 2(1977), 193-222.

[8] K. Dharmendra, Semilinear elliptic problems with singular terms on the Heisenberg group, Complex Var Elliptic Equ. 2019, doi.org/10.1080/17476933.2018.1557157

[9] J.I. Diaz, J. M. Morel, L. Oswald, An elliptic equation with singular nonlinearity. Commun. Part. Differ. Equ. 12(1987), 13331344.

[10] A.V. Lair, A.W. Shaker, Entire solution of a singular semilinear elliptic problem, J. Math. Anal. Appl. 200 (1996), 498-505.

[11] A. V. Lair and A. W. Shaker, Classical and weak solutions of a singular semilinear elliptic problem, J. Math. Anal. Appl. 211(1997), 193-222.

[12] A. C. Lazer and P. J. Mckenna, On a singular nonlinear elliptic boundary-value problem. Proc. Am. Math. Soc, 111(1991), 721-730.

[13] C. D. Luning, W. L. Perry, An iterative method for solution of a boundary value problem in non- Newtonian fluid flow, J. Non-Newton. Fluid Mech. 15(1984), 145-154.

[14] W.L. Perry, A monotone iterative technique for solution of $p^{\text {th }}$ order $(p<0)$ reaction-diffusion problem in permeable catalysis, J. Comput. Chem. 5(1984), 353-357.

[15] G. Stampacchia, Le probléme de Dirichlet pour les équations elliptiques du second ordre à coefficients discontinus, Ann. Inst. Fourier (Grenoble) 15(1965), 189-258.

[16] C.A. Stuart, Existence and approximation of solutions of nonlinear elliptic equations, Math. Z. 147 (1976), 53-63.

[17] K.O. Widman, Inequalities for the Green function and boundary continuity of the gradient of solutions of elliptic differential equations. Math. Scand. 21(1967), 17-37.

[18] B. Xuan, The solvability of quasilinear Brezis-Nirenberg-type problems with singular weights, Nonlinear Anal. Theory Methods Appl, 62(2005) 703-725.

[19] Z. Zhang, J. Cheng, Existence and optimal estimates of solutions for singular nonlinear Dirichlet problems, Nonlinear Anal. 57 (2004), 473-484. 